\title{
Potential Damage to Flight Hardware from MIL-STD-462 CS02 Setup
}

\author{
Patrick K. Harris, P.E., NCE \\ ManTech Advanced Systems International \\ Aerospace Technology Applications Center \\ Goddard Space Flight Center, Code 540.5 \\ Greenbelt, MD 20771 USA \\ paharris@pop700.gsfc.nasa.gov
}

\author{
Nathan F. Block, NCE \\ ManTech Advanced Systems International \\ Aerospace Technology Applications Center \\ Goddard Space Flight Center, Code 540.5 \\ Greenbelt, MD 20771 USA \\ nblock@pop700.gsfc.nasa.gov
}

\begin{abstract}
The MIL-STD-462 CS02 conducted susceptibility test setup includes an audio transformer, with the secondary used as an inductor, and a large capacitor. Together, these two components form an L-type low-pass filter to minimize the injected test signal input into the power source. Some flight hardware power input configurations are not compatible with this setup and break into oscillation when powered up. This, in turn, can damage flight hardware. Such an oscillation resulted in the catastrophic failure of an item tested in the Goddard Space Flight Center (GSFC) Large electromagnetic compatibility (EMC) Test Facility.
\end{abstract}

\section{Keywords}

Electromagnetic Compatibility, Testing, Measurements

\section{INTRODUCTION}

During preparations for MIL-STD-462 CS02 testing of the InfraRed Array Camera (IRAC) Warm Electronics Assembly (WEA) at the NASA GSFC Large EMC Test Facility on Dec. 28, 1999, the device under test (DUT) broke into oscillation when powered up. This oscillation occurred prior to application of the CS02 test signal (1.0 $\mathrm{V}_{\mathrm{RMS}}$ from $50 \mathrm{kHz}$ to $400 \mathrm{MHz}$ ). The oscillation damaged the DUT flight hardware and caused a catastrophic failure on a flight printed circuit board. This required that a nonflight board be substituted to complete the remaining test sequences. Investigations were initiated to determine the cause of the oscillation, which was determined to be the result of a $1 \mathrm{mH}$ inductance in series with the power line. This inductance is present on the secondary of a Solar Electronics Co. Model 6220-2 audio transformer used in conjunction with a parallel $7,000 \mu \mathrm{F}$ capacitor. Together, these two components form an L-type low-pass filter to minimize the injected test signal input into the power source (see Figure 1). In this configuration, the transformer primary winding is left open circuited.

\section{RESULTS OF INVESTIGATION}

As stated previously, investigations determined the cause of the oscillation on the IRAC WEA, which was the result of the $1 \mathrm{mH}$ inductance on the secondary of the audio transformer in series with the power line. Measurements showed that the inductance value was $0.8 \mathrm{mH}$ at $1 \mathrm{kHz}$ with a Q of 10 . The primary of the audio transformer is open circuited, as per typical MIL-STD-462 CS02 test setup (see Figure 1). Contacting the audio transformer manufacturer, Solar Electronics Co., confirmed the potential oscillation problem and they suggest terminating the primary of the transformer with a $2-\Omega$ resistor that will in turn reduce the secondary inductance to less than $200 \mu \mathrm{H}$. (A short on the primary reduces the inductance on the secondary to $7 \mu \mathrm{H}$.)

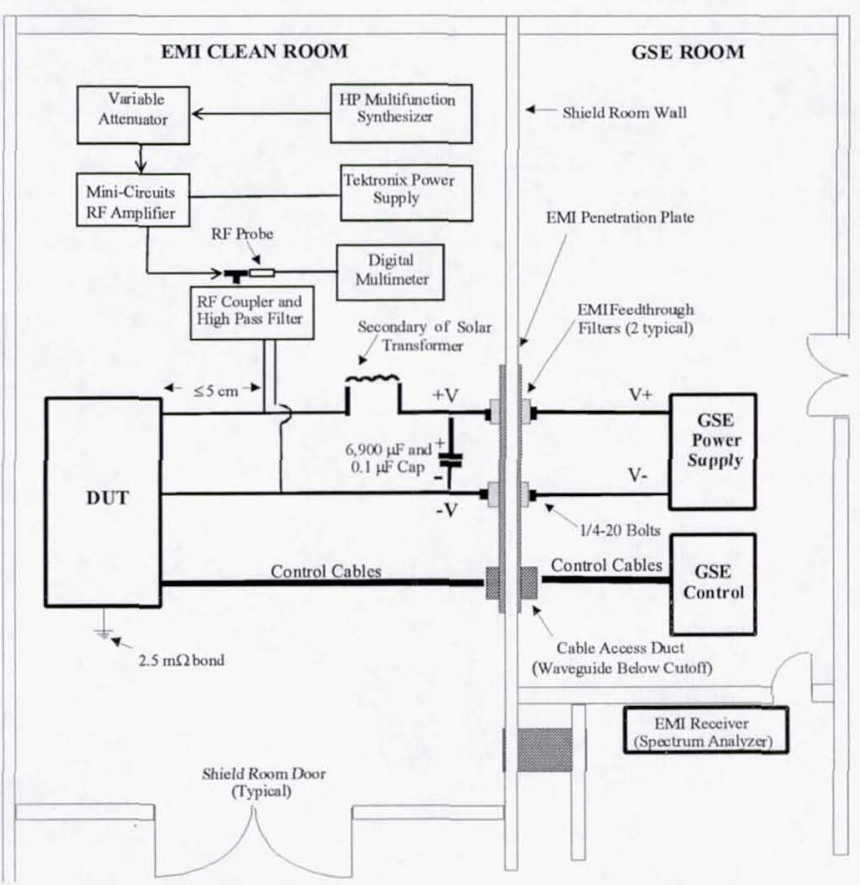

Figure 1. CS02 Test Setup in Large EMC Test Facility

The IRAC WEA power supply design uses Interpoint hybrid power converters. The "power on" sequence of the converters is controlled by circuitry that holds the inhibit pin low until converter turn-on is desired. This circuitry is powered directly from the spacecraft $28 \mathrm{~V}$ supply and is located on the load side of the input power filter. The absolute maximum voltage rating for this circuitry is $40 \mathrm{~V}$. These integrated circuits were destroyed when the peak voltage on these circuits reached $50 \mathrm{~V}$ during the 
oscillation. The oscillation was believed to have occurred because the DUT power supply was turned on with the conducted susceptibility test transformer open circuited (typical MIL-STD-462 CS02 setup). This resulted in added inductive impedance being inserted in series with the power supply. This impedance caused the oscillation in the series circuit made up of the laboratory supply, the audio transformer and the IRAC WEA power converters. This oscillation can occur in some instances, depending on the power converter design. It should be pointed out that the oscillation occurred without application of the CS02 test signal. In addition, test facility personnel repeatedly warned the IRAC project personnel about the oscillation, yet they continued to remain powered up, which lead to the circuitry damage.

\section{CORRECTIVE ACTION FOR EMC TESTS}

The IRAC WEA Interpoint power converters have a switching-mode power supply with a negative input resistance at low frequencies. This negative-input resistance in combination with the L-type low-pass EMI filter (which lies between the source and the switchingmode power supply), under certain conditions, can constitute a negative resistance oscillator, and is the source of the WEA's instability [1]. Preferably, a suitable filter should be used with the switching-mode power supply. Unfortunately, the EMI filter is fixed per MIL-STD-462 specifications. However, knowledge of the Interpoint power converter design permits criteria developed by Middlebrook to be applied that will ensure not only system stability but also have virtually no effect on loop gain, output impedance and line rejection. The Middlebrook criterion requires the output impedance of the EMI filter to be less than the open-loop input impedance of the switching-mode power supply as shown in Figure 2.

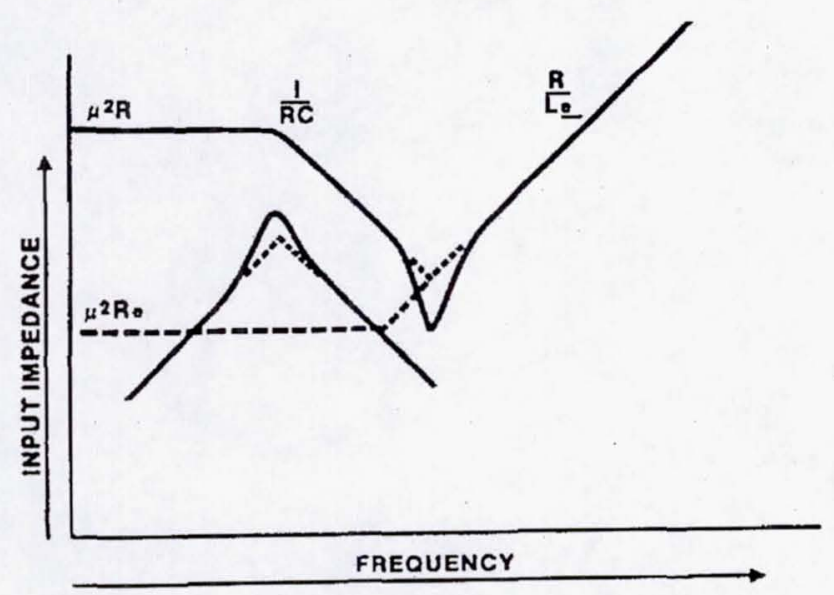

Figure 2. Middlebrook Stability Criterion

The top curve represents the switching-mode regulator open-loop input impedance, $\mathrm{Z}_{\mathrm{i}}$, which is the reflected impedance of the series-resonant loaded averaging (output) filter. The lower solid curve represents the EMI input filter's output impedance, $Z_{\mathrm{s}}$, in the single-section low-pass filter configuration. However, in real-world conditions, the output impedance configurations are more complex. A twosection filter is smaller and lighter than a single-section filter with the same peaking, power loss and attenuation at the switching frequency. In addition, the power system (including the generator and the distribution network) is a part of the impedance configuration. The Middlebrook criterion applies to DC systems and to AC systems in which the rectifying diodes are in continuous conduction. However, since discontinuous line current results in increased rectification harmonics, it is recommended to avoid this by using inductive input filters rather than capacitive ones.

The addition of the input filter impairs the performance feature of the regulator. In other words, a nonzero $Z_{s}$ (Figure 3) lowers the loop gain, raises the line transmission function factor (this opens a frequency window to pass the input noise to load), and raises the regulator's output impedance (the lower the output impedance, the better the regulation). The loop gain and line transmission are essentially unaffected if the criterion for stability discussed is met-the output impedance of the input filter is very much less than the input impedance of the regulator. However, it is necessary that the input filter's output impedance be well below the dotted line in Figure 2 for the regulator's output impedance to be essentially unaffected.

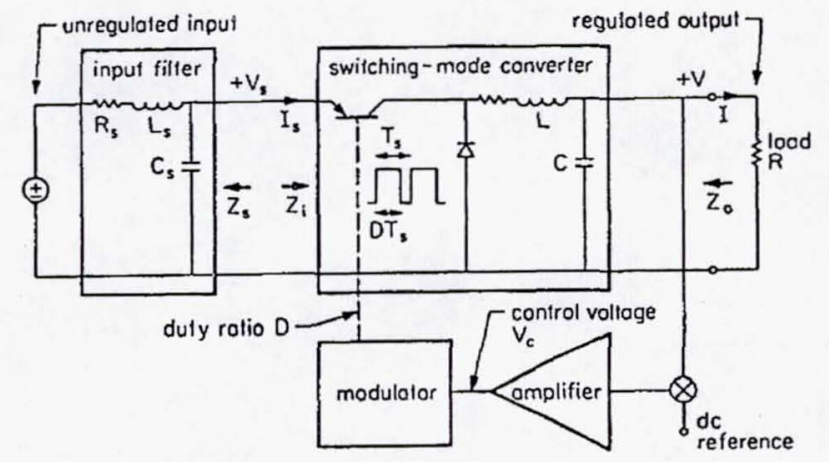

\section{Figure 3. General Switching-Mode Regulator Configuration}

MIL-HDBK-241B recommends two design considerations to meet or exceed the stability criterion:

1. Keep the resonant frequency or frequencies of the EMI filter far away from the effective resonant frequency of the regulator's output filter; preferably using a lower resonance for the EMI filter thereby simultaneously decreasing the EMI conducted to the input power lines.

2. Lower the Q of either or both the EMI and output filters preferably by lossless damping. Where Q is: 


$$
\mathrm{Q}=\mathrm{R}_{\mathrm{d}} / \mathrm{R}_{\mathrm{o}}
$$

Where:

$$
\mathrm{R}_{\mathrm{o}}=\mathrm{L} / \mathrm{C}
$$

$R_{d}$ is the shunt damping resistance. $R_{0}$ is the characteristic resistance of the filter.

The inductor used in the NASA GSFC Large EMC Facility is a Solar Electronics Co. 6220-2 audio transformer. As stated previously, the inductance value measured at $1 \mathrm{kHz}$ is $0.8 \mathrm{mH}$ with a $\mathrm{Q}$ of 10 . A $2.5-\Omega$ resistor, which should reduce the chances of resonance caused by the inductance of the transformer, is now being used at GSFC for CS02 and CS06 testing. The inductor used in the NASA GSFC Small EMC Facility is a Solar Electronics 6220-1A audio transformer. The inductance value measured at $1 \mathrm{kHz}$ is $1.26 \mathrm{mH}$ (see Figure 4(a)). The same fix (a 2.5- $\Omega$ resistor across the primary winding) is also being used for this transformer (see Figure 4(b)). (The inductance achieved corresponds with the $25 \mu \mathrm{H}$ series value specified by Duff [2], who points out that the U.S. Army's Notice 3 to MILSTD-462 requires a LISN instead of the series inductor). These corrective actions have been implemented to avoid any recurrence of oscillations during future EMC testing.

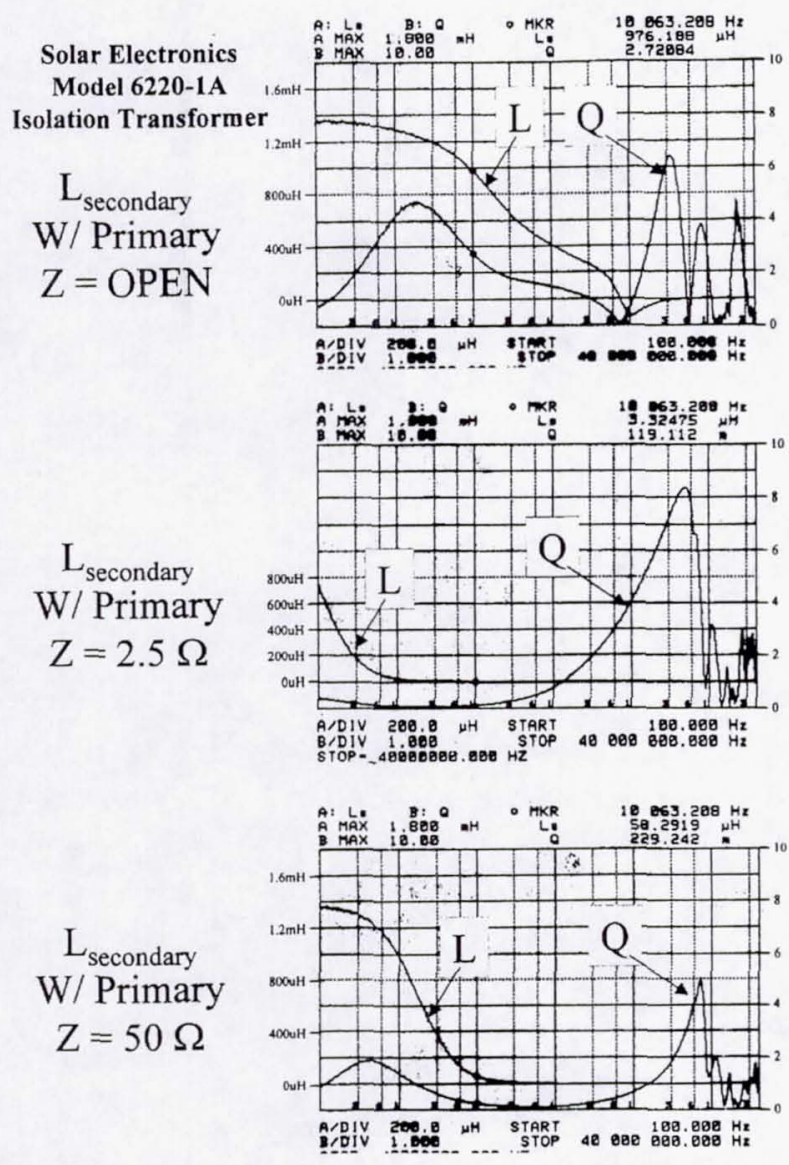

(a)

Figure 4. Solar 6200-1 Transformer Characteristics
It should be noted that the application notes from Solar Electronics Co. show the 6220-1A transformer primary not terminated with a resistor, which could lead to the potentially damaging test setup. These application notes were written before this problem began to surface at other EMC test facilities. Contacting Solar Electronics Co. confirmed that the termination should be used. It should also be pointed out that the oscillation problem would not occur in MIL-STD-462D/E CS114 testing (which replaced $\mathrm{CS} 02$ ) because of differences in the test setup.

The oscillations noted may not always occur under conducted susceptibility test conditions, but standard practices should be observed to insure that it never occurs again. EMC test procedures should highlight the importance of connecting the audio transformer to the test signal audio power amplifier and powering on the amplifier before power is applied to the DUT for CS01 testing, which uses the low-pass EMI filter. In this test, the test signal is injected directly into the primary of the audio transformer, as shown in Figure 5, instead of leaving it open circuited. If the DUT is turned on before the test signal Solar audio power amplifier, an open circuit condition will result. (The output circuit of the Solar Model 6220-1A power amplifier is open if the line power switch is in the off position.) Also, the test signal audio power amplifier should only be turned off after it has been confirmed that the DUT power has been turned off at the completion of a test.

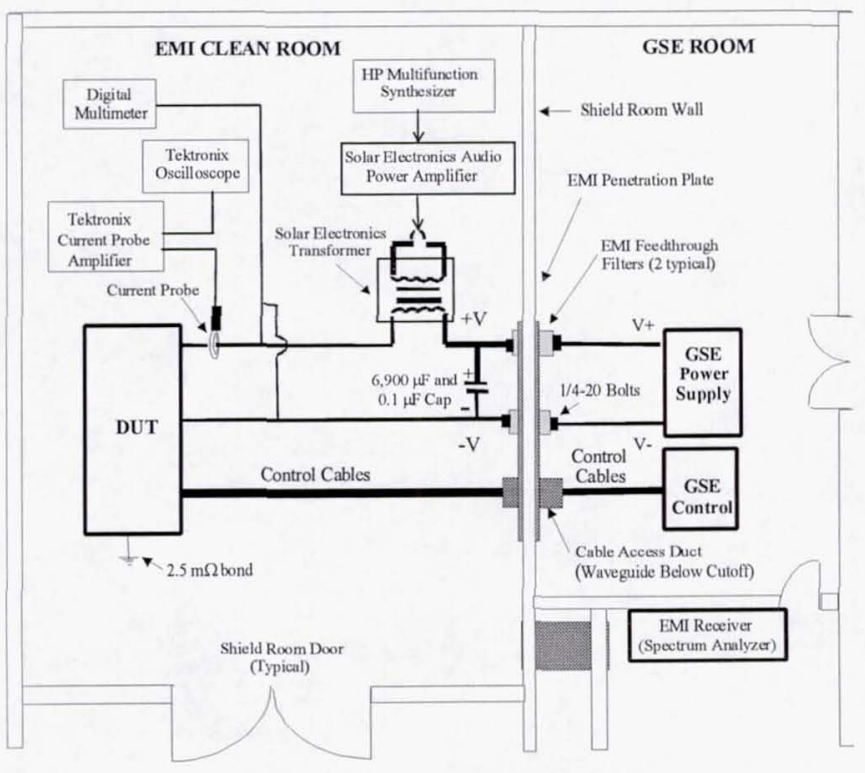

Figure 5. CS01 Test Setup in Large EMC Test Facility

\section{CONCLUSION}

In order to avoid any future oscillation occurrences, EMC test procedures should be updated to indicate clearly that the primary side of the audio transformer must be loaded down with the test signal power amplifier and that this amplifier must be turned on prior to DUT turn-on for CS01 
testing. This will avoid having a high inductance (in the order of several $\mathrm{mH}$ ) on the secondary winding in series with the power line. Furthermore, NASA engineers suggest that the primary side of the transformer always contain a $50-\Omega$ resistor across its windings to avoid ever having high inductance on the secondary side regardless of whether or not the primary side is connected to a powered on amplifier. Hence, two protection schemes exist to prevent any recurrence of the oscillations on power lines during conducted susceptibility EMC testing. However, Figure 4 (c) shows that the $50-\Omega$ resistor does not significantly reduce the inductance until approximately $10 \mathrm{kHz}$, thus the $2.5-\Omega$ resistor is preferred. In addition, this resistor value is close to the $2-\Omega$ value suggested by the audio transformer manufacturer.

\section{ACKNOWLEDGEMENTS}

This paper describes work performed under National Aeronautics and Space Administration (NASA) contract NAS5-32537.

\section{REFERENCES}

[1] MIL-HDBK-241B, Design Guide for Electromagnetic Interference (EMI) Reduction in Power Supplies, Revision B, U.S. Department of Defense, Sept. 30, 1983.

[2] Duff, W., Fundamentals of Electromagnetic Compatibility, Volume 1, Interface Control Technologies, Gainesville, VA (1988). 